\title{
Appropriate selection of a mouse strain in accordance with the vascular properties
}

\author{
Takuya Kishi ${ }^{1}$
}

Received: 24 May 2020 / Revised: 11 June 2020 / Accepted: 11 June 2020 / Published online: 27 July 2020

(c) The Japanese Society of Hypertension 2020

In experimental animal research on hypertension, various mouse models have been widely used in this decade [1]. Compared to other animal models, such as rats or rabbits, mouse models can be easily genetically manipulated, and inbred mice show genetic homogeneity. Moreover, a telemetry system for monitoring arterial pressure is available in mouse models, and continuous arterial pressure in a conscious state for a long period can be assessed in mice [1]. However, different mouse strains can exhibit significant genetic divergence [2], and arterial pressure also varies among different mouse strains [3]. The cardiac structure and function are different among various mouse strains. The widely used strain $\mathrm{C} 57 \mathrm{Bl} / 6 \mathrm{~J}$ was reported to maintain better cardiac function than other inbred strains after cardiac ischemia [4]. Echocardiographic, exercise response, and telemetric variables can vary between strains, and C57B1/6J mice are known to have eccentric cardiac hypertrophy and increased exercise tolerance [5]. In the pathophysiology of these differences, vascular stiffness should be the focus. Previous studies have demonstrated that vascular stiffness is a predictor of cardiovascular mortality and major cardiovascular events [6] and that vascular stiffness is determined by complex alterations to both the composition of and the interactions between the constituent cellular and structural elements of the vessel [7]. Vascular stiffness assessed by pulse wave velocity (PWV) correlates independently with poor outcomes [6]. As reported in many previous studies, Steppan et al. indicated that evaluation of vascular stiffness is an essential first step to develop targeted therapies [8]. These previous studies strongly indicated that we should select a mouse model in accordance with the properties of

Takuya Kishi

tkishi@iuhw.ac.jp

1 Department of Graduate School of Medicine (Cardiology), International University of Health and Welfare, Okawa, Fukuoka, Japan the mouse strain. However, the variability of vascular stiffness indices has not been fully assessed in mouse strains.

In this context, Steppan et al. reported an impressive study, "Commonly used mouse strains have distinct vascular properties", in Hypertens Res [9]. These researchers focused on the mouse strain-dependent differences in key vascular stiffness indices among frequently used inbred mouse strains (C57B1/6J, 129S, and B16/129S). The vasocontractile response was determined with a phenylephrine dose-response analysis, endothelium-dependent responses were determined with acetylcholine, and endotheliumindependent vasorelaxation was investigated by using sodium nitroprusside. PWV was measured noninvasively by using a high-frequency, high-resolution Doppler spectrum analyzer. Elastic properties were analyzed by tensile testing. The major elastic vessel showed distinct properties in different strains and within male and female animals. C57B1/6J mice had the most compliant aortas and exhibited greater phenylephrine-induced vasoconstriction and acetylcholine-induced vasorelaxation responses than the other strains. C57Bl/6J mice had thinner vessel walls with fewer lamellar units than the other strains, whereas $129 \mathrm{~S}$ mice had the thickest walls with the most lamellar units. However, in contrast to the passive stiffness, PWV, arterial pressure, and heart rate were similar in all of the groups. Overall, the low passive vascular compliance of the thin vessels was offset by high contractility and higher active tone, resulting in similar in vivo vascular stiffness. These results suggest that the response of $\mathrm{C} 57 \mathrm{Bl} / 6 \mathrm{~J}$ mice to hypertensive stimuli would be distinct from that of the other two strains (129S and B16/129S).

The most important implication in Steppan's study was that the underlying properties of different mouse strains are distinct, despite superficial similarities in arterial pressure. These differences among mouse strains cannot be disregarded when choosing a mouse strain for cardiovascular studies. We selected a control wild-type mouse that closely matched the homozygous knockout mouse. The present study clearly 
showed that C57B16 mice have the highest dynamic range for vasoreactivity and stiffness studies. However, $129 \mathrm{~S}$ mice are a poor choice for vasoreactivity and stiffness studies. As mentioned by Steppan et al., the ideal study design would use a het/het breeding strategy that would yield wild-type and homozygous littermate mice. In cases where mouse models have been bred to homogeneity, the use of the same background strain as control wild types is critical. Moreover, we should realize that the vascular stiffness was different between males and females in the present study. Experimental studies using mice must be verified in more than one background strain and in both genders to maximize the robust interpretation of preclinical data.

It has already been established that vascular stiffness is a hallmark of aging and hypertension [6]. Vascular stiffness is also highly predictive of major adverse cardiovascular events, such as perioperative myocardial infarction, heart failure, and death [6]. Furthermore, vascular stiffness is closely related to age-associated hypertension, which precedes the development of clinical hypertension [8]. To assess the pathophysiology of hypertension, researchers have conducted many experimental studies using mice [10-15], which will be increased. We know that different mouse strains demonstrate a certain degree of genetic variation and differences in blood pressure, and mouse strains that have identical normotensive blood pressures at baseline should be selected.

As described by the authors, a limitation of the present study was that arterial pressure was measured by the tail cuff method. Further studies with continuous arterial pressure measurement by telemetry system are necessary.

In conclusion, Steppan et al. showed that the underlying vascular properties of different inbred wild-type mouse strains (C57B1/6J, 129S, and B16/129S) are distinct despite superficial similarities in arterial pressure. As noted by the authors, we should carefully identify the proper controls for each genetic modification and to verify the conclusions in more than one mouse strain to minimize the risk of false positive results.

\section{Compliance with ethical standards}

Conflict of interest The author declares no conflict of interest.

Publisher's note Springer Nature remains neutral with regard to jurisdictional claims in published maps and institutional affiliations.

\section{References}

1. Wilde E, Aubdool AA, Thakore P, Baldissera L Jr, Alawi KM, Keeble J, et al. Tail-cuff technique and its influence on central blood pressure in the mouse. J Am Heart Assoc. 2017;6:e005204.

2. Doevendans PA, Daemen MJ, de Muinck ED, Smits JF. Cardiovascular phenotyping in mice. Cardiovasc Res. 1998;39:34-49.

3. Schlager G, Weibust RS. Genetic control of blood pressure in mice. Genetics 1967;55:497-506.

4. Barnabei MS, Palpant NJ, Metzger JM. Influence of genetic background on ex vivo and in vivo cardiac function in several commonly used inbred mouse strains. Physiol Genomics 2010;42A:103-13.

5. Hoit BD, Kiatchoosakun S, Restivo J, Kirkpatrick D, Olszens K, Shao H, et al. Naturally occurring variation in cardiovascular traits among inbred mouse strains. Genomics 2002;79:679-85.

6. Vlachopoulos C, Aznaouridis K, Stefanadis C. Prediction of cardiovascular events and all-cause mortality with arterial stiffness: a systematic review and meta-analysis. J Am Coll Cardiol. 2010;55:1318-27.

7. Steppan J, Barodka V, Berkowitz DE, Nyhan D. Vascular stiffness and increased pulse pressure in the aging cardiovascular system. Cardiol Res Pract 2011;2011:263585.

8. Steppan J, Wang H, Bergman Y, Rauer MJ, Tan S, Jandu S, et al. Lysyl oxidase-like 2 depletion is protective in age-associated vascular stiffening. Am J Physiol Heart Circ Physiol. 2019;317: H49-59.

9. Steppan J, Jandu S, Wang K, Kang S, Savage W, Narayanan R, et al. Commonly used mouse strains have distinct vascular properties. Hypertens Res. 2020; https://doi.org/10.1038/s41440-0200467-4 (Epub ahead of print).

10. Ehara Y, Hirawa N, Sumida K, Fujiwara A, Kagimoto M, OokiOkuyma $\mathrm{Y}$, et al. reduced secretion of parathyroid hormone and hypocalcemia in systemic heterozygous ATP2B1-null hypertensive mice. Hypertens Res. 2018;41:699-707.

11. Yin X, Cao H, Wei Y, Li HH. Alteration of the IL-33-sST2 pathway in hypertensive patients and a mouse model. Hypertens Res 2019;42:1664-71.

12. Okuyama Y, Hirawa N, Fujita M, Fujiwara A, Ehara Y, Yatsu K, et al. The effects of anti-hypertensive drugs and the mechanisms of hypertension in vascular smooth muscle cell-specific ATP2B1 knockout mice. Hypertens Res. 2018;41:80-7.

13. Mukohda M, Fang S, Wu J, Agbor LN, Nair AR, Ibeawuchi SC, et al. RhoBTB1 protects against hypertension and arterial stiffness by restraining phosphodiesterase 5 activity. $\mathrm{J}$ Clin Invest. 2019;129:2318-32.

14. Gao L, Zuo Z, Tian J, Ali Q, Lin Y, Lei H, et al. Activation of SIRT1 attenuates klotho deficiency-induced arterial stiffness and hypertension by enhancing AMP-activated protein kinase activity. Hypertension. 2016;68:1191-9.

15. Lin JR, Zheng YJ, Zhang ZB, Shen WL, Li XD, Wei T, et al. Suppression of endothelial-to-Mesenchymal Transition by SIRT (Sirtuin) 3 alleviated the development of hypertensive renal injury. Hypertension. 2018;72:350-60. 